\title{
Young Learners: How advantageous is the early start?
}

\author{
Koutsompou Violetta-Irene (M.A) \\ ${ }^{1}$ (Department of English Language and Literature, Empire State College/ State University of New York, New \\ York College, Athens, Greece)
}

\begin{abstract}
This article's main purpose is to inform you about the importance of starting a foreign or second language at a young age. The title of the article is actually a question "How advantageous is the early start?" and that's a controversial issue concerning the fields of education and linguistics. Parents, teachers, professors, scientists and linguists have tried to give a definite answer to the above question but they still have not achieved to come up with a conclusion. This article will basically inform you not only about the advantages of starting young a language and how children react, behave and perceive the world but also will present the disadvantages of starting young and the beliefs which exist in most countries but will mostly give attention to Greece. Furthermore, we will turn to specialized fields such as neurolinguistics, psychology, applied linguistics and education in order to gain knowledge from the research which has conducted up until now and will help us draw our own conclusions. Generally, at the end of the article it is my belief that the reader will get a clear picture of what is happening with this issue and will also realize the significance of knowing all this information because it is our duty to educate properly our children and equip them with knowledge that will carry on for the rest of their lives; knowledge which will help them create a better future not only for themselves but also for the next generations and society.
\end{abstract}

Keywords: acquisition, education, Greece, psycholinguistics, young learners

\section{INTRODUCTION}

This article is entitled "Young Learners: How advantageous is the early start?" and really this is an issue of great concern since it has to do with the future of our children and their knowledge. This article will basically try to inform you about children as learners and provide you with the necessary tools in order to draw your own conclusions. According to the findings of this article which were examined here, concerning issues such as age, motivation, the brain processes and various other themes, we will have the chance to understand how the above factors played either a negative or a positive role in acquiring $L_{2}$ and we will realize the importance of starting at a young age a foreign language.

\section{What Do We Mean By Early Start?}

First of all, we should begin with the word "childhood" and its meanings. The word "childhood" varies from country to country. In many parts of the world kids take on "adult" responsibilities at ages when in other countries they are still protected within their schools. These differences will guide to differences in the purposes of language learning. Likewise, attitudes to authority, to teaching and to learning generally vary from culture to culture. However, we can list a number of characteristics which young learners share. In the first place, young learners are merely now starting their schooling, so that teachers have an important chance to mould their expectations of life in school. As a group they are potentially more differentiated than secondary or adult learners, for they are closer to their varied home cultures and new to the conventionality increasingly required across cultural groupings by the school. Apart from this, children usually have a tendency to be dedicated and excited learners, without the inhibitions which older children bring to their schooling. Moreover, their learning can be intimately associated with their development of thoughts and concepts, for the reason that it is so close to their first experience of formal schooling. To conclude with young learners, we mention that they also want physical movement and action as much as inspiration for their thinking, and as we must say that the closer together these can be the better results we will have in the end. ${ }^{1}$ After defining the term "childhood" and presenting the basic characteristics that the majority of primary level learners usually share, now we will make an effort to define what we mean when we talk about early start. Early start is related to the age of the learner and as we can understand age is an important factor and of course plays a major role in learning a language. When we talk about early start, basically, we mean what is the most appropriate age for a child to start learning a foreign language. There is a widely-held belief that younger learners do better than older learners. This is supported by the critical period hypothesis according to which there is a fixed span of years during which

\footnotetext{
${ }^{1}$ Brumfit et al.,1997, p.v
} 
language learning can take place naturally and easily and after which it is not probable to be totally successful, of course this will be examined extensively in another part of this project. Studies of learners in naturalistic learning situations provide the most convincing evidence that the younger the better. From the above we understand that age is an important aspect in the field of second language acquisition and plays a special role in the process of learning a language. So, early start is strongly connected with age or let's put it in another way, age and early start is the same thing and more specifically in this research we give the obvious picture that when we say early start we mean the age of the child. Last, but not least, judging from the above, we summarize with the following: the sooner the better, meaning that children should start at a young age learning a second language in order to benefit from the procedure and acquire as much as possible from the foreign language. We will realize the importance of age in the following sections of this project so you will appreciate better the above.

\section{HistoricAl REVIEW}

There has been an upsurge in the last few decades as far as the English language is concerned. English has become a lingua franca; it is a language which is learned in all over the world because it is an easy one comparing it with other languages for example Greek or Chinese. Everyone can communicate through English and it is very important especially for the percentage of people who are involved in trade. If we go back in time, for example twenty years ago, we will realize that people did not give much attention in the English language. In Greece, few people were interested in English and most of them lived in big cities such as Athens; in villages there was a completely different situation because back then they were keen on professions like teacher, lawyer or peasants. Furthermore, in those years the only university which promoted its certificates was the University of Cambridge which it is thought that it is the most prestigious one still today. Moreover, the material was very limited because there was no interest in foreign languages. Nowadays, things have changed rapidly due to globalization which played a basic role in the development of the English language. In Greece, a significant step is that English has been introduced in public schools as a required lesson for children and there has been an increase in foreign language institutes known as frontistiria in the Greek market and all that due to the increased interest from the people. Moreover, the books concerning young learners come out every year in many different colors and each of these books offers different things and at the end you do not know which book to purchase for your child. In addition to the above, we have to mention that in the Greek market many English and American universities promote their degrees and the people literally 'run' to give and basically pass these examinations and get a certificate because a young person must know English in order to find a job.Apart from the above, we observe that the last few years there is a revival of interest in the teaching to young learners. After much interest in the 1960s, the period of communicative language teaching saw a concentration on adults, with relatively little concern for primary level activity but now this is changing. Young learners are now in the center of the education and much emphasis is given on them. Massive material for teachers on how to teach young learners and many seminars are held in order to improve children's language skills. The most impressive part is that universities in the States and in England have created especially designed master degrees on teaching English to young learners, for instance the University of York has done a master's degree with a title like the one which was given above. As we understand from the above there is a concentration on young learners and to teach them effectively and this is very important due to the fact that this change is a result of the research conducted in the past few decades concerning issues such as second language acquisition or neurolinguistics which revealed that we should focus on children because their brain is like a sponge which keeps everything inside and we should take advantage from that and find ways to help children learn as much as possible easily. Now, we will move on to the next section in which we will learn about the human brain and its procedures.

\section{THE HUMAN BRAIN}

There is an intimate link concerning language and the brain. Specific areas of the brain are devoted to language and damage to these areas disrupts language. After much research conducted in this field has proved that injury, removal or any kind of problem in the left hemisphere can cause severe consequences for language development especially in children. According to the above findings scientists have also come up with the conclusion that normal brain development depends on early and regular exposure to language. But let's have a closer look in the complex system called brain; the brain is roughly organized like a peach in that there is a large outer layer, the cerebrum, surrounding an inner kernel, the brainstem, which keeps people alive. The brain divided in two parts, the cerebral hemispheres, one on the right and one on the left connected like Siamese twins. The former controls the left side of the body and the latter the right. One of them, usually the left, is the more powerful dominant hemisphere. This is not only due to the ability it has to control the right side of the body - and the majority of humans are right-handed- but also because it normally controls language. In the early part of the nineteenth century Franz Joseph Gall put forth theories of localization, which means that different human abilities and behaviors were noticeable to specific parts of the brain. It was not until 1861 that language 
was specifically related to the left side of the brain $^{2}$. At a scientific meeting in Paris, Paul Broca stated that we speak with the left hemisphere on the basis of his finding that damage to the front part of the left side, now called Broca's area, had as a consequence loss of speech while damage to the right hemisphere did not. Language, then, is said to be lateralized. Lateralization is the term used to refer to any cognitive functions which are primarily localized to one side of the brain or the other. ${ }^{3}$ Nowadays, with such damages in Broca's area are said to suffer from Broca's aphasia. Aphasia is the neurological definition used to refer to language problems that follow brain lesions caused by a stroke, a gunshot wound, or infection. The basic problems that patients who have Broca's aphasia face are the following: labored speech, disturbed word order, omission of grammatical morphemes such as articles, prepositions etc., and also word-funding pauses; for instance, a patient said "yes-ah-Monday ah-dad-and dad-ah-hospital-and ah-.....ah girl-and gums and I". Moreover, as we understand Broca's aphasics show syntactic deficits. In 1873, Carl Wernicke presented a research paper in which he described another kind of aphasia that revealed that patients who suffer from this variety have the following characteristics: speak fluently with fine pronunciation and intonation but with many lexical errors, phonological ones and difficulty in comprehending speech. And as a result this area in the back portion of the left hemisphere is known as Wernicke's area and the patient are said to have Wernicke's aphasia. An example is "I can't talk all of the things I do, and part of the part I can go alright, but I can't tell from the other people". Below there is given a photo of the brain presenting both areas:

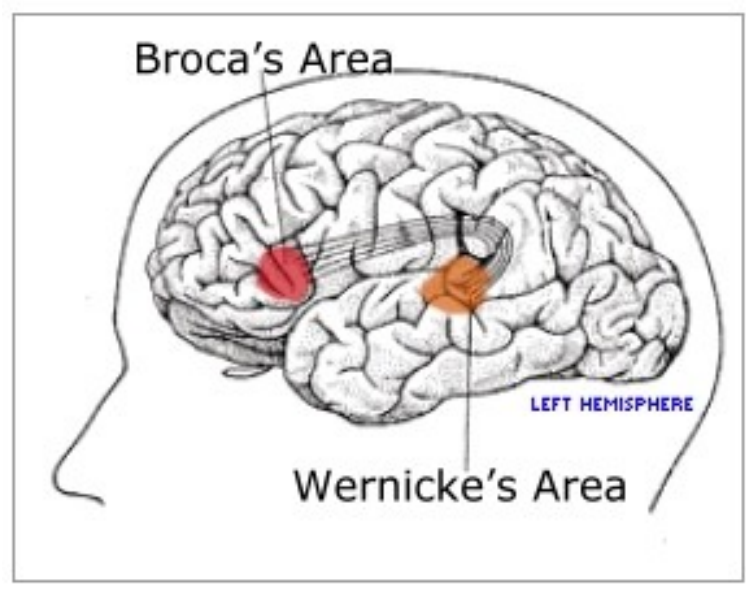

According to the above information, there is no doubt that people who have problems with speech production such as agrammatic aphasics basically have injuries towards the front of the brain whereas those who have problems with comprehension such as fluent aphasics have injuries towards the back.

\section{The Critical Period And Famous Cases}

Let's return to language and more specifically how a child can acquire language. Under normal conditions, a child is introduced to language almost at the moment of birth. Adults talk to the child and to each other in the presence of him/her. Children do not have need of explicit language instruction but they do need contact with the language in order to develop normally. Children who do not receive linguistic input during their early years in life do not accomplish native like grammatical competence. Both behavioral tests and brain imaging studies have shown that late exposure to language alters the primary organization of the brain for language. The Critical Age Hypothesis ${ }^{6}$ is a division of the biological basis of language and states clearly that the ability to learn a native language within a fixed period, meaning from birth up until puberty. The period is referred to as critical period. During this critical period, language acquisition proceeds easily, quickly, and without external involvement. Furthermore, studies have proved that after this stage the acquisition of grammar is difficult and for some people never fully achieved. Children deprived of language during this period demonstrate atypical patterns of brain lateralization. It is widely believed from scientists that during childhood the human brain is most ready to receive and learn a particular language. If a child does not acquire language at that specific time for any number of reasons then, this child, unfortunately, will face a great difficulty to learn language later on. A large percentage of children in recent years, due to environments of extreme social isolation

\footnotetext{
${ }^{2}$ Notes provided by Professor Lambrini Philippatos

${ }^{3}$ Ibid footnote 2

${ }^{4}$ Yule, 2004, p.168

${ }^{5}$ Ibid footnote 4

${ }^{6}$ Fromkin et al., 2003, p.52
} 
were unable to adapt fully the language, these examples constitute, confirm and also give insight into what happens when lateralization process takes place without an accompanying linguistic input.

At this point we will present some notable examples in order to realize the importance of the situation. In 1758 Carl Linnaeus first included Homo ferus_(wild or feral man) as a subdivision of Homo sapiens. According to Linnaeus, a defining characteristic of Homo ferus was the lack of speech or evident language of any kind. In 1920 two feral children, Amala and Kamala, were found in India hypothetically having been reared with wolves. A notable case, which is also known from Truffaut's film THE WILD CHILD, is the one with a child named Victor who was left in the woods at a very young age, was found in 1798 and finally succeeded to survive; this boy is also known as "the wild boy of Aveyron". ${ }^{7}$ Now, we should take a look to another important case study concerning language acquisition. A distinguished one, which came to the light of publicity in 1970, is that of Genie, a 13-year-old who had spent most of her life tied to a chair locked in a small room. Her father was intolerant of any kind of noise and had beaten Genie whenever she made a noise. Moreover, there had been no radio or television and the child's only human contact was forbidden, even her mother was not allowed to spend more than a few minutes with her, only long enough to feed her. Genie had spent her whole life in a state of physical, sensory and psychological deprivation. ${ }^{8}$ As a conclusion, Genie was unable to use language, but when she was brought into care she began to respond trying to imitate sounds and communicate. She was able to gain knowledge of a large vocabulary, including colors, shapes and objects but her syntax remained simple and morphology never fully developed. The linguist Susan Curtiss who worked with her for many years reports that "the stringing together of content words, often with rich and clear meaning, but with little grammatical structure". "Genie started learning language after the critical period and unfortunately she was never able to fully acquire the grammatical rules of English; moreover, it should be mentioned that many utterances produced by her at the age of 15 were not different from those produced by agrammatic aphasics. None of these children, due to isolation, was able to speak or knew any language at the moment of reintroduction to the social environment. Another important case which also should be mentioned is that of a deaf woman called Chelsea, in California. ${ }^{10}$ Doctors wrongly diagnosed her as retarded and as a result she never attended school but fortunately a neurologist, after many years, diagnosed her real problem so Chelsea received extensive language therapy and was able, little by little, to acquire a large vocabulary but, just like Genie, could not develop grammar. A study of the localization of language in her brain showed an equivalent reply to language in both hemispheres.

\section{ACQUISITION AND LEARNING}

"Passing hence from infancy,...I was no longer a speechless Infant, but a speaking boy... it was not that my elders taught Me words...in any set method... when they named any thing, And as they spoke turned towards it, I saw and remembered that They called what they would point out, by the name they uttered." Saint Augustine's account in the $4^{\text {th }}$ century of his own history of language acquisition. ${ }^{11}$

Some researchers distinguish between acquisition and learning. According to Yule ${ }^{12}$ the term "acquisition" refers to,

".... the gradual development of ability in a language by using it naturally in communicative situations" on the other hand, "learning ... applies to a conscious process of accumulating knowledge of vocabulary and grammar of a language." For instance, for a Greek pupil English is a language which will be learnt at school with the teacher and through activities that will improve students' skills in order to communicate with a foreigner; also, it is taught as a separate lesson in the classroom. As a result, the child gains knowledge of the language. In contrast, acquisition has to do with "spontaneous learning" meaning that a child will acquire language through interaction and everyday communication with native speakers. For example, in order to acquire English one should go for long periods of time in England or to the States or another solution is to move there; thus, the child will not only be proficient in the four skills but also will acquire the accent of that country. It's worth remembering that Rod Ellis ${ }^{13}$ in his book "The Study of Second Language Acquisition" states that acquisition "... refers to the subconscious process of "picking up" a language through exposure; on the other hand, learning is related to the conscious process of studying it."

\footnotetext{
${ }^{7}$ Ibid footnote 6

${ }^{8}$ Yule, 2004, p. 171

${ }^{9}$ Fromkin et al., 2003, p.53

${ }^{10}$ Ibid footnote 9

${ }^{11}$ Bloom, 1993, p. 84

${ }^{12}$ Yule, 2004, p. 175

${ }^{13}$ Ellis, 2003, p. 14
} 
A similar division between "learning" and "acquisition" is made by Wolfgang Klein ${ }^{14}$ states that the former is connected to the guided learning and the latter is basically, referring to spontaneous learning. According to the above opinions, it is probable for learners for to acquire or to learn rules independently and at separate times. Judging from the above information we conclude that learning ought to be seen as an imitative rather like the domestication of a natural procedure. In order to understand better the distinction between acquisition and learning as well as realize the vital role of the critical period, we will examine L1 (First or Mother Language) and L2 (Second Language) and their differences. An example, which shows how L1 is acquired, may help here. Now, imagine a young child aged one year old, interacting with her mother. Usually, children around this age produce one word at a time. Think that the mother leaves the room and after a few minutes returns with the child's favorite doll, the child says doll, not mother or mummy. Later, when the mother is helping her with lunch the child points at the milk and says more. After a while, the child is struggling with her shoes and the mother asks her what she is doing, the simple response is off. The general conclusions that arise from these observations are: firstly, the child might be familiar with, at least in an implicit way, some of the rules of language to use words properly. We could say that she uses more not as an imitation or an isolated word but as a request in order to make her mother understand that she wants her mother to bring the milk closer. Secondly, the word doll is less clear, meaning that the child might be making an observation on her personal environment by labeling a toy she finds interesting or on the other hand, she may be requesting the doll. The question is: how do we determine what she is trying to say? One possible way to see what happens is if the mother does nothing. The probable reaction is that if the word were meant as a request, the child might become more insistent and the most common way to show that is by pointing at the doll and by repeating the word doll; whereas if it were meant as a comment, the child's behavior should end with mother's mere acknowledgment of the object. Hence, the child has learned certain pragmatic rules to guide her choice of words. Finally, it appears that children know more than they say. It's important to mention that first language acquisition is significant for the speed with which it takes place usually during infancy. Infants experience words as one part of a complex event. When the child hears a word or a phrase or a sentence this, let's say, word is entered in memory along with other perceptual and personal data about its circumstances of use. The data for these associations consist of the perpetual and functional contingencies experienced with the word and these can be an action by the child or someone else, as well as an object in the context. As a result the speech unit is one element of an episode connected with other elements such as objects, actions and persons. ${ }^{15}$ Finally, we come up with the conclusion that all developmental accounts of a child's first words have noted the strong connection between word and object, word and action, or word and event. In order for a child to learn a language he/she must hear that language being used. Of course, the child receives messages since he/she grows up in a language-using environment called family. Parents interact with their child and in turn the child responds to messages they send. Basically, the child uses the parrot-fashion method to acquire the language; our little creature is consistently imitating adults, from parents to anyone one can think. The child repeats words, expressions, grammar and through this procedure he/she succeeds to adopt a lot of vocabulary from their speech. To quote Yule ${ }^{16}$ :

"The speed of acquisition and the fact that it generally occurs, without overt instruction, for all children, regardless of great differences in a range of social and cultural factors, have led to the belief that there is some 'innate' predisposition in the human infant to acquire language."

A child's vocabulary moves beyond fifty distinct words when the toddler is around eighteen to fifteen months; we observe that the child improves the words he/she knows and goes a step further, tries to combine them, for instance baby chair, cat eat, mommy good. Adults understand what the child wants to say due to the context of the utterance. In addition, a child both communicates and confirms that the utterance worked. By the age of two and a half the child's vocabulary is increasing quickly and by three it has developed to hundreds of words and pronunciation has become closer to the form of the adult language. As well as by the age of five the child has accomplished to complete the larger part of the fundamental language acquisition process. ${ }^{17}$ For this reason, when a child enters elementary school has become a great language user this can be justified especially if we think that he/she can easily communicate orally with adults and also have the ability to provide arguments in a discussion he/she finds interesting. Judging from the above, many scientists draw the conclusion that the child is in a good position to start learning a second (or in other words foreign) language. In contrast, most educational systems prefer not to introduce foreign language in the classroom until much later. The question which arises from that is: why learning a second language is so hard when the acquisition of the mother language is clear-cut?

\footnotetext{
${ }^{14}$ Klein, 1997, p.20

${ }^{15}$ Bloom, 1993, p. 83

${ }^{16}$ Yule, 2004, p.175

${ }^{17}$ Yule, 2004, p.186
} 


\section{Second Language Acquisition}

In order to answer this question we have to discover how second language learning works. First of all, we have to define what second language is. The term "second" is generally used to refer to any language other than the first language. ${ }^{18}$ How can a child acquire a second language? A second language can be acquired in a variety of ways, at any age, for different purposes, and to varying degrees. Therefore, we could make a division of different types of second language learning. Generally, a basic difference has been made between tutored and untutored (spontaneous) language learning. ${ }^{19}$

The term "spontaneous learning" is used to indicate the acquisition of a second language on a daily basis communication, in a realistic environment and independent from systematic guidance. The term "guided learning" refers to learn a language through teaching, meaning exercises, tests, grammar, listening, speaking and reading texts and all that with the guidance of a teacher. At this stage, we should mention Krashen's theor $y^{20}$ in relation to spontaneous and guided learning. The vital point of the theory is that learning in this sense is always affected through a 'monitor' or in other words an attempt on the part of the learner to manage his language output and to self-correct it at whatever time necessary. The monitor controls the learner's language knowledge in the same way as a rider controls a horse. The monitor can become useful in a communication situation only in the following cases: firstly, there is enough time to operate it. Secondly, the speaker is concerned with the correctness of his speech production, and thirdly, the speaker knows the correct rule. As we can understand the theory underlines the difference between guided and spontaneous language acquisition. In both cases, however, the crucial part is acquisition in the sense of a subconscious process governed by certain rules. The learning (and indirectly, teaching) in which the learner is involved exerts, to a certain extent, a controlling influence upon the 'acquisition' of the language.SLA theory has endeavored to apply the same principles to people learning a second language, even to adult learners, arguing that if the focus is function and meaning, form will be acquired effortlessly and naturally. ${ }^{21}$ Monolingual approaches to language teaching are the natural outcome of such assumptions and of the additional assumption that the acquisitional environment of the first language can in some way be reproduced in second language acquisition. If these assumptions were accurate, a monolingual approach would make sense. The question is: are they correct and accurate? Brumfit ${ }^{22}$, amongst others, argues that the acquisition of a second language differs from that of the first language, in that first language acquisition is in some sense the instantaneous development of the ability of language as well as of the structure of a particular language. Furthermore, it is apparently a natural and automatic product of the process of socialization with adults.

\section{Why Learning A Second Language Has Become A Controversial Issue?}

Learning a second language, particularly in a classroom situation, is not such a natural or automatic process. The learner already has a language that allows him to communicate. To ask him/her to give up that language and return to some pre-linguistic stage of development is not particularly realistic._Most people attempt to learn another language during their teenage or adult years in few hours each week of school time and by spending some others in private foreign language schools (for example, Greece) rather than via continuous contact experienced by a child, with lots of other occupations in contrast to children that have little to do and with an already known language available for most of their daily communicative requirements.

Moreover, they face problems such as cannot cope with the new sounds of another language for example French or Japanese, they cannot pronounce easily words, they generally have an accent and they may make syntactic or morphological mistakes that are unlike errors of children acquiring their first language. L2 users usually make word order errors, more specifically, early in their development, as well as morphological ones in grammatical gender and case. Of course, they tend to fossilize ${ }^{23}$ and unfortunately, no amount of teaching or correction can undo this situation. With some exceptions, adults do not simply pick up a second language. It usually requires careful concentration, if not intense study and memorization, to become proficient in a second language. Once again with the exception of some remarkable individuals, adult second-language learners do not often accomplish native like grammatical competence in the L2. So, we can conclude that age is a significant factor in developing L2 acquisition and of course, once again, we should mention that the critical period is strongly related to age and by extension not only to the acquisition of the first language but also plays a vital role in the acquisition of the second language, respectively.

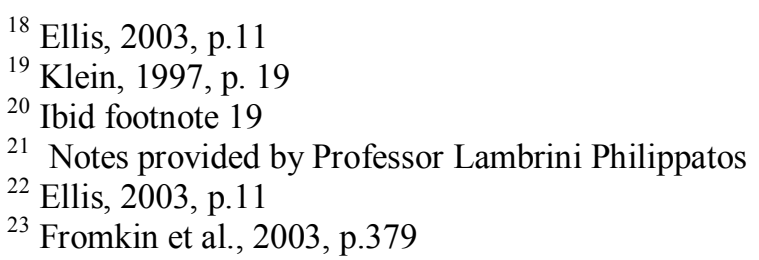




\section{Advantages Of Starting L2 At A Young Age}

According to Piaget ${ }^{24}$ the child is seen as frequently interacting with the world around him/her, solving problems that are presented by the setting. It is through taking action to solve problems that learning occurs. Piaget gives a much less vital role to language in cognitive development than does Vygotsky. It is action, rather than the development of the first language which, for Piaget, is essential to cognitive development. Piagetian psychology differentiates two ways in which development can take place as a consequence of activity: assimilation and accommodation. The former happens when an action takes place without any change to the child and the latter involves the child adjusting to features of the environment in some way. Both of the above are originally adaptive processes of actions but they turn out to be process of thinking. Accommodation is an important suggestion that has been taken into second language learning under the label 'reconstructing', used to refer to the re-organization of mental representations of a language. From a Piagetian perspective, a child's thinking develops as slow growth of knowledge and intellectual skills towards a final stage of proper, rational thinking. Though, gradual growth is punctuated with specific basic changes, which cause the child to pass through a series of stages. At each stage, the child is competent of some types of thinking but still incapable of others. Particularly, the Piagetian endpoint of development - thinking that can influence formal abstract categories using rules of logic - is held to be unavailable to children before they reach 11 years of age or more. Vygotsky's ${ }^{25}$ views of development differ from Piaget's in the importance he gives to language and to other people in the child's world. The development of the child's first language in the second year of life is held to produce a fundamental move in cognitive development. Language provides the child with a new tool, opens up new opportunities for doing things and for organizing information through the use of words as symbols. Young children can often be heard talking to themselves and organizing themselves as they carry out tasks or play, in what is called private speech. As children get older they speak less and less aloud, and distinguish between social speech for others and 'inner speech', which continues to play an important role in regulating and controlling behavior.

Vygotsky placed great emphasis on the importance of social interaction in cognitive development and more specifically in the development of language and thinking. On the whole, Vygotsky saw the child as first doing things in social environment, interacting with other people and how language is helping in many different ways and steadily changing and going away from reliance on others to independent action and thinking. Now, let's go back and present the advantages of starting a foreign language in the early years of life. There is a significant discuss about whether children learn language better or more competently than older learners or adults. Generally speaking the data is uncertain and vague. A large percentage of people are in favor of the claim that it's better to start at a young age. First of all, young children have more opportunities than adults because they don't have other responsibilities but their only occupation is to learn and study. Second of all, they are learning all the time without having the worries and responsibilities of adults. Their parents, friends, and teachers help them in learning. We all know that children are curious and ask always "why?" even if they know the answer; this process's result is that it helps them learn the world. Moreover, they have a physically powerful individual need to learn if they are surrounded by people speaking the second language, and there are strong social pressures to do what their elders expect. Furthermore, the brain is more adaptable before puberty than after, and that acquisition of languages is probable without self-consciousness at an early age. Another advantage is that children have less negative attitudes to foreign languages and cultures than adults, and as a consequence they are better motivated than adults. In addition, children's language learning is more intimately incorporated with real communication because it depends more on the direct physical environment than does adult language. And that is truth because an adult is afraid of expressing his opinion on an issue in a foreign language due to his fear that he/she may make a mistake and become embarrassed in the end but children do not realize this and they express freely themselves without worrying about the correct way of saying something. Another point worth mentioning is that children dedicate enormous quantities of time to language learning, compared with adults and they are better for the reason that they do more of it. ${ }^{26}$ And finally, learners who start as children achieve a more native like accent than those who start as adolescents or adults.

A notable study is that conducted by Oyama ${ }^{27}$ in 1976 who investigated 60 male immigrants who had entered the United States at ages ranging from 6 to 20 years and had been resident there for between 5 and 18 years. She asked two adult native speakers to judge the nativeness of the learners' accents in two 45 second extracts taken from performance on a reading-aloud task and a free-speech task. Oyama reports a very strong effect for 'age of arrival' but almost no effect for 'number of years' in the United States. She found that the youngest arrivals performed in the same range as native speaker controls. Other studies which have investigated

\footnotetext{
${ }^{24}$ Cameron, 2005, p.3-7

${ }^{25}$ Ibid footnote 24

${ }^{26}$ Brumfit et al, 1997, p.vii

${ }^{27}$ Ellis, 2003, p.489
} 
the effects of age on pronunciation support the younger is better position. Similar results have been obtained for the acquisition of grammar. As Singleton ${ }^{28}$ writes:

"Concerning the hypothesis that those who begin learning a second language in childhood in the long run generally achieve higher levels of proficiency than those who begin in later life, one can say that there is some good supportive evidence and that there is no actual counter evidence".

As a result we come up with some general conclusions, presented below, which unite the pieces of the puzzle and we get a clearer picture of the role of age in second language: Firstly, only child learners are able of acquiring a native accent in informal learning contexts. Long puts the critical age at 6 years but Scovel argues for a pre-puberty start. Singleton points out those children will only acquire a native accent if they receive vast exposure to the second language. Secondly, children perhaps are more likely to acquire a native grammatical competence. Thirdly, children are more likely to accomplish higher levels of achievement in both pronunciation and grammar than adults. Lastly, the procedure of acquiring second language grammar is not significantly affected by age, but that of acquiring pronunciation definitely is. ${ }^{29}$ Furthermore, there are a number of reasons for teaching English at primary level that do not rely simply on the claim that is the best time to learn languages well. Below we list the reasons ${ }^{30}$ :

$\checkmark$ The necessity to expose children from an early age to an understanding of foreign cultures so that they grow up broadminded and sympathetic to others.

$\checkmark$ The need to connect communication to the understanding of new concepts.

$\checkmark \quad$ The need for maximum learning time for important languages-the earlier you start the more time you get

$\checkmark$ The advantage of starting with early second language instruction so that later the language can be used as a medium of teaching.

Judging from the above conclusions and findings we come up with the view that there is no evidence to suggest that teaching foreign languages to young children actually produces bad results as well as the age factor in language acquisition conclude that an early start does give advantages and certainly does not do any harm; in contrast, it helps children to become familiar with the culture which produced the language, become more open minded and encourages the child to want to learn more and more about the language. But before we summarize and reach any definite conclusion we must go a step further and also examine the disadvantages of starting at a young age.

\section{Disadvantages- The Other Side Of The Coin}

Many people claim that if a child starts a foreign language in his/her early years not only will not benefit from it but also he/she will become confused by the exposure to it. Of course, this point of view is not accurate because in many countries such as Africa and Asia children learn two foreign languages at the same time. In addition, published information on the outcomes of early language learning come from the North American experience with immersion of teaching, where native speakers of English are placed in Frenchspeaking nursery and infant schools and vice versa ${ }^{31}$. Learning a second language through immersion differs from learning a foreign language as a subject lesson numerous times a week. When we talk about immersion we mean that students study at school subjects through L2 and therefore have contact and more experience with the language. In such contexts, young learners who have an early start expand and uphold advantages in some areas of language skills. ${ }^{32}$ But here we are going to examine the methods used up until now in the language classroom and to show how these methods influence young learners to learn a language. Another important disadvantage, which must be mentioned here, is the lack of appropriate methods used in the language classroom for many years. Approaches designed to promote L2 learning in this century have the tendency to reflect many different opinions on how a foreign language is best learned. But before describing the methods, we will define what an approach is and what a method is. When we talk about approaches in the language teaching we mean the theories about the nature of language and language learning that serve as the sources of practices and principles in the language teaching. ${ }^{33}$ So, an approach explains how people acquire their knowledge of the language and makes statements about the circumstances which will support successful language learning. And when talking about method refers to the practical realization of an approach. The originators of a method have arrived at decisions about types of activities, roles of teachers and learners, the kinds of material which will be helpful, and some model of syllabus organization. Methods include various procedures and techniques as part of their

\footnotetext{
${ }^{28}$ Ellis,2003, p.490

${ }^{29}$ Brumfit et al., 1997, p. vi

${ }^{30}$ Ibid footnote 29

${ }^{31}$ Cameron, 2005, p. 17

${ }^{32}$ Ibid footnote 31.

${ }^{33}$ Harmer, 2001, p.78
} 
standard fare. ${ }^{34}$ After defining approaches and methods in the language teaching now we are going to focus on the methods used in the last few decades in the language classroom.

\section{Grammar-Translation Method}

This is the most traditional approach to teach a second or foreign language and this method has its roots in the traditional approach to the teaching of Latin. Long lists of words and a set of grammatical rules have to be memorized and the written language rather than the spoken is emphasized. ${ }^{35}$ This method didn't help L2 learners because after learning the language through this way a student leaves the classroom without actually know the language. For example, learners leaving school, having achieved high grades in French class via this method, typically find themselves at a loss when confronted by the way the French in France actually use their language. ${ }^{36}$

\section{Audiolingual Method}

This is a very different approach which became popular in the 1950s and basically emphasizes the development of the spoken language. This involved a systematic presentation of the structures of the L2, moving from the simple to the more complex, often in the form of drills which the student had to repeat. ${ }^{37}$ This approach was strongly related to a belief that the fluent use of a language was basically a set of 'habits' which could be developed with a lot of practice. Much of this practice included hours spent in a language laboratory repeating oral drills. The following example shows a typical Audio-lingual drill ${ }^{38}$ :

Teacher: There's a cup on the table...repeat

Students: There's a cup on the table

Teacher: spoon

Students: There's a spoon on the table

Teacher: book

Students: There's a book on the table

This method is still popular today and many foreign language institutes follow this policy by using versions of the audiolingual method which is still very common in language teaching but of course this method is not the only one they use in the classroom. The critics of this approach claim that isolated practice in drilling language patterns bears no resemblance to the interactional nature of actual language use. ${ }^{39}$ Furthermore, this method becomes boring for both the teacher and the student

\section{Communicative Approaches}

The Communicative approach or Communicative Language Teaching (CLT) is the name which was given to a set of beliefs which included not only a re-examination of what aspects of language to teach but also a shift in emphasis in how to teach. A guiding principle was to train students to use language forms appropriately in a variety of contexts and for a variety of purposes. The general belief is that plentiful exposure to language in use and plenty of opportunities to use it are vitally important for a student's development of knowledge and skill $^{40}$. Activities in CLT typically involve students in real or realistic communication where accuracy of the language they use is less important than successful achievement of the communicative task they are performing. What matters in these activities is that students should have a desire to communicate something. In other words such activities should replicate real communication. The conclusion is that, according to this method, students must communicate in the context of real, everyday situations.

\section{General Conclusions}

The method which will be used in the language classroom is very important because can affect students either negatively or positively. The method and the way the teacher reacts and behaves are two of the most basic reasons for a student to love the language or hate it forever. A large percentage of people in Greece do not like English due to the method used in the language institute they attended or because of the behavior of the teacher. At this point we should say that motivation is an important factor for students to learn the language. It has been noted that those who experience some success are among the most motivated to learn. ${ }^{41}$ Motivation comes from

\footnotetext{
${ }^{34}$ Ibid footnote 33

${ }^{35}$ Yule, 2004, p.193

${ }^{36}$ Ibid footnote 35

${ }^{37}$ Ibid footnote 36

${ }^{38}$ Harmer, 2001, p. 79-80

${ }^{39}$ Yule, 2004, p.193

${ }^{40}$ Harmer, 2001, p. 84-85

${ }^{41}$ Yule, 2004, p.195
} 
outside and from inside. Extrinsic motivation is caused by any number of outside factors, for example, the need to pass an exam, the possibility of a future travel or the hope of financial reward. Intrinsic motivation comes from within the individual. Therefore, a person might be motivated by the enjoyment of the learning process itself or by a desire to make themselves feel better ${ }^{42}$. As we understand motivation is crucial for a person in order to learn so teachers and by extension the methods they use must motivate students to learn the language. Now, we are going to focus on learning disabilities and how can these influence negatively a child to learn a foreign language; I would also like to add how important it is to diagnose a learning difficulty from the early years in order to avoid psychological problems and also help the child improve himself/herself.

\section{Learning Difficulties}

Another important issue of great concern is that children face learning difficulties in acquiring a foreign language. The most common learning difficulty which many children in our days face is dyslexia. Dyslexia is a difficulty in which a child cannot read, write and remember easily. Nobody quite knows what causes dyslexia at the moment. There has been a genuine increase in the amount of research taking place, and a number of possibilities are beginning to come out, but the waters are still quite gloomy. The overall picture is that dyslexia can be caused in two ways: (a) by inherited factors and (b) by hearing problems at an early age. If someone can find out the problem at the very beginning the situation can be faced with various solutions. Sometimes a child has experienced early hearing problems and has also inherited genes which dispose him/her towards difficulties dealing with the printed word. These children are often found to be quite severely dyslectic and need a lot of support through their school and college years. As a general rule, it is widely believed that there is a variety of types of dyslexia but there is no pattern which all dyslectic children and adults fit. Some dyslectic children have good short-term memory while others are weak in this area and some of these children have a 'talent' in sciences such as math and biology. Up to this point we have to say that there are some learning strategies which can make a huge difference.

Learning strategies to overcome the difficulties related to dyslexia can make an important difference, as mentioned above, to the performance of a dyslectic child or adult. More specifically, the well known method called the 'multi-sensory' which is very helpful and involves teaching children to learn spellings not only by hearing and saying the sounds of the letters, but also by using their visual and tactile memories by writing letters in the air or on the carpet making them with plasticine. ${ }^{43}$ Through this procedure dyslectic learners become even more creative and this helps them a lot. On the whole, it is crucial for children to start learning a foreign language at a young age because such learning difficulties can be found out easily in these age groups and can be faced with many solutions. If parents or the child realizes it after childhood the situation becomes very complicated and needs even more effort in order to achieve good results.

\section{Recommendations-Personal Opinion}

After searching and studying about the issue of whether start at a young age a foreign language or not I should admit that at the beginning of the report I was really confused with the information gathered and I was not convinced from the one side or the other in order to come up with a conclusion. Of course, after reading again and again and spending hours speculating I decided to analyze, think rationally and critically the evidence from both sides. My personal opinion is that children should start a foreign language at their early years because this will help them a lot due to the fact that everything that they will learn is spontaneous and with repetition will stick in their memory and by extension they will use it in their communication; another advantage is that they will not realize that they actually have class but they will enjoy contact with the language because they will find it funny and unusual. Children love repetition and that's the way they learn their mother language by hearing the elders and repeat their words (parrot fashion). Besides this we should mention that children do not care if they make mistakes because they are more interested in communicating with others. For all these reasons and for many more which have been explained in this project I strongly believe that the sooner a child starts to learn a foreign language the better it is for him/her. As a conclusion, I would like to say that judging from the findings of the questionnaires as well as from the interviews with the teachers of English I think that people who are involved in the field of teaching should be informed and should keep up to date because things change and we have to be prepared in order to face every situation.

\section{Conclusion}

As a conclusion, I would like to express my feeling of satisfaction and personal fulfillment due to the great experience I had doing this research concerning issues of importance such as first and second language acquisition, neurolinguistics, psychology and young learners in the fields of linguistics and education.

\footnotetext{
${ }^{42}$ Harmer, 2001, p.51

${ }^{43}$ ELT News, Learning Difficulties: Dyslexia, November 2003
} 
Fortunately, I had the opportunity to conduct in this article as well as to present my own thoughts and ideas about the advantages of starting a foreign language from a young age. I would also like to add that it was very useful and a pleasure for me to observe the way other people think about second language as well as studying and researching in an issue of great concern in the field of language. Finally, I hope the above issues presented in this project have been adequately explained and I want to feel that I offered something in the field of linguistics and education. Last, but not least, I honestly expect that the people who are going to read this article will benefit from it and will become more interested in these issues.

\section{REFERENCES}

\section{Books}

[1]. Bloom, Lois. The Transition from Infancy to Language. Cambridge University Press, 1993.

[2]. Brumfit, Christopher. Moon, Jayne. \& Tongue, Ray. Teaching English to Children. Longman, 1997.

[3]. Butterworth, George \& Harris Margaret. Principles of Developmental Psychology. Psychology Press Ltd, Publishers, 1998.

[4]. Cameron, Lynne. Teaching Languages to Young Learners. Cambridge University Press, 2001.

[5]. Ellis, Rod. The Study of Second Language Acquisition. Oxford University Press, 2003.

[6]. Fromkin, Victoria. Rodman, Robert \& Hyams Nina. An Introduction to Language. United States of America: Heinle, 2003: $7^{\text {th }}$ edition.

[7]. Harmer, Jeremy. The Practice of English Language Teaching. Longman editions. England, 2001.

[8]. Klein, Wolfgang. Second Language Acquisition. Cambridge University Press, 1997.

[9]. Nunan, David. Research Methods in Language Learning. Cambridge University Press, 1997.

[10]. Yule, George. The Study of Language. Cambridge University Press. United Kingdom, 2004.

[11]. Wade, Carole \& Tavris, Carol. Psychology. Pearson Education. New Jersey, 2003: $7^{\text {th }}$ edition.

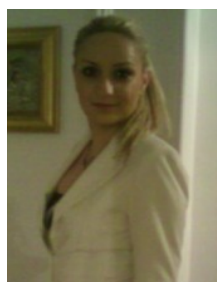

Ms Koutsompou Violetta-Irene holds a BA in English and Psychology (2007) from the State University of New York, USA, a Masters degree in English and Comparative Literature (2009) from the University of Indianapolis, USA, an accredited Certificate in TEFL (2007) and a Diploma in Counseling Psychology (2013) from City Unity College, Athens, Greece. She is currently working on an MA in Counseling Psychology with the City University of Seattle, USA. Her areas of interest and concentration are psychology of child development, children's literature and its impact on the cognitive development, depression, grief and loss, stress management, second language acquisition, postmodern literature, women's psychology and writings, book editing and writing. She has worked in private language institutions since 2005; she was interested and worked with children with special learning difficulties such as dyslexia, as well as with children with behavioral problems in the language classroom. She is currently conducting a qualitative research concerning postnatal depression. She is married and has one child. 